\title{
Deconstruyendo prejuicios: discursos e imaginarios sobre el homosexual en Cartagena (1970-1977)
}

\author{
Muriel Jiménez Ortega ${ }^{1}$ \\ Universidad de Cartagena
}

\section{Resumen}

En el trabajo que presentamos a continuación nos proponemos develar la forma en que los prejuicios y la actual homofobia tienen una trascendencia histórica, enmarcada en la forma como hemos significado a los sujetos a través de discursos producidos durante años (discursos que han llevado a que se naturalice, normalice e institucionalice la discriminación y la exclusión en sus formas más violentas contra los homosexuales). Analizaremos para ello la construcción histórica y discursiva del homosexual en Cartagena desde los saberes psiquiátricos y jurídicos que representan a este sujeto en la prensa publicada en la primera mitad de la década de los 70 .

Palabras clave: homosexualidad, homofobia, discursos, imaginarios, prensa.

\begin{abstract}
This paper we present next proposes to reveal homophobia prejudices of today and its transcendence historic, framed in the way like we have given meaning to the Subjects for years (the speeches have naturalized, standardized and institutionalized the exclusion and discrimination at their most violent forms against homosexuality). We will analyze the historic construction and discursive to the homosexual at Cartagena since psychiatric and judicial knowledge which represent this Subject in published press in first half at 70's.
\end{abstract}

Key words: homosexuality, homophobia, speeches, imaginaries, press.

1 Historiadora de la Universidad de Cartagena. Candidata a Magíster en Estudios de la Cultura con Mención en Políticas Culturales de la Universidad Andina Simón Bolívar (Quito-Ecuador). Joven Investigadora de Colciencias, 2011-2012. Miembro del Grupo de Estudios Literarios y Representaciones del Caribe (Gelrcar), semillero de Investigación de CEILIKA (categoría A, Colciencias). Este artículo hace parte de la investigación presentada como requisito para optar al título de Historiadora de la Universidad de Cartagena. e-mail: gotikdeth@hotmail.com

Recibido: Marzo, 2010 - Aprobado: Junio, 2010. 


\section{Introducción}

Desde hace algunos años, gracias a los derechos garantizados en la Constitución de 1991 y a los recientes fallos de la Corte Constitucional para las parejas del mismo sexo -en cuanto a derechos patrimoniales, seguridad social y vivienda, entre otros-, pareciera que en Colombia se ha logrado una igualdad desde los estamentos del Estado con las parejas heterosexuales que mantienen uniones de hecho. Lo curioso es que, en medio de estos derechos otorgados al sector LGBT (lesbianas, gays, bisexuales, transgéneros), la situación de exclusión y discriminación cotidiana no ha cesado. Continúa más viva que nunca la violencia simbólica y los crímenes de odio contra un cuerpo homosexual rechazado, abyecto y que es un "otro" diferente a las mayorías heterosexuales

Así, por más que en nuestro país se estén dando cambios en la legislación civil y penal -lo cual ha conducido a una discriminación menos oficial-, esto no quiere decir que hayan acabado los prejuicios en la realidad social; y esto lo demuestra el hecho de que cuanto más visible se hace la homosexualidad, más aumenta la violencia homofóbica. Este panorama apunta, ciertamente, a que la homofobia se sigue reproduciendo en la cotidianidad -en la práctica- y está fuertemente ligada a la forma cómo se ha construido históricamente al homosexual como un sujeto sexual abyecto, anormal, desviado, enfermo, transgresor y pecador.

Estas páginas pretenden abordar al hombre homosexual, sujeto cuya atracción sexual, emocional, sentimental y afectiva está dirigida a individuos de su mismo sexo. Es decir, seguimos la inscripción de la homosexualidad dentro del terreno de las identidades sexuales, cuestión muy distinta a su identidad de género. Como señala Marta Lamas (1995: 61-79), la identidad no es únicamente constituida en lo social, sino que está definida como un papel, rol, estatus, grupo de referencia o de pertenencia, y es también una formación psíquica. Es allí donde se definen los conceptos de identidad genérica, como el sentimiento de pertenecer al género masculino o femenino, y la identidad sexual, como el posicionamiento del deseo de una persona ya sea homosexual o heterosexual.

Nos centraremos aquí en el hombre homosexual, porque sus caracterizaciones están atravesadas por las transgresiones a los roles de género, muy a pesar de que la homosexualidad implica un tipo de identidad sexual, como dijimos anteriormente, y no una identidad 
de género (que aplicaría para las personas transgénero) ${ }^{2}$. A lo largo de este trabajo, sin embargo, el género - definido como "el sistema de relaciones de poder constituido en la producción discursiva, mediante la que se establece la naturalidad de los sexos, se definen las relaciones entre los mismos y se designa normativamente la heterosexualidad como destino de la sexualidad" (Muñoz, 2006: 108)-, nos será de gran ayuda como categoría transversal. El género establece, en efecto, unos roles socialmente construidos en donde el hombre sustenta el poder y las relaciones de dominación. De éste se espera que cumpla con el designio de ser masculino, padre proveedor y ante todo, heterosexual. En este sentido, el sujeto homosexual rompe y transgrede los fundamentos reproductores del patriarcado y a la vez la posición de dominio dentro de las lógicas sociales del género.

Cabe decir que, hasta el momento, en la ciudad de Cartagena no existen investigaciones sobre este tema en específico: muy pocos historiadores reconocen en el cuerpo y la sexualidad problemas de tipo histórico, y es el nuestro un primer acercamiento a un campo de estudio hasta ahora poco atendido: la historia de las ciencias y la historia de la sexualidad en el contexto de Cartagena. Las siguientes líneas abordan así los distintos discursos que circularon entre 1973 y 1977 en Cartagena, reveladores de un trasfondo en los saberes psiquiátricos y jurídicos que, para el periodo de estudio, ayudaron a construir imaginarios de exclusión sobre el homosexual en Cartagena. Dividiremos para ello nuestro trabajo en tres momentos: en el primero, mostraremos cómo algunos historiadores han abordado la problemática del sujeto homosexual; en el segundo, analizaremos la patologización del homosexual, y en el tercero, evidenciaremos la manera en que el discurso psiquiátrico y el discurso jurídico se complementan y se manifiestan en la prensa.

\section{Una historia de las sexualidades no normativas}

La historia de la sexualidad en Occidente ha contado con importantes investigaciones e historiadores como Phillipe Aries, Michel Foucault y Jean-Louis Flandrin Si bien Historia de la sexualidad I: La voluntad del saber, de Foucault, no es una historia propiamente sobre la homosexualidad, establece conceptos y formas de estudiar la sexualidad desde los dispositivos de poder -referencia importante

\footnotetext{
${ }^{2}$ No nos centraremos en la homosexualidad femenina debido a la poca o nula documentación que nos permite referirnos a ella en este periodo y a través de un estudio de la prensa cartagena de la década de los setenta (esto como producto de la invisibilizacion histórica que ha existido sobre la sexualidad de la mujer en Occidente y en la ciudad).
} 
para llevar a cabo una historia sobre las formas de saber y poder sobre la homosexualidad-. Nos dice, por ejemplo, que el siglo XIX se inaugura con una proliferación de discursos, por parte de la medicina, la psiquiatría y la justicia penal, cuya finalidad era sustentar una única verdad sobre la sexualidad legítima: la hetero-reproductiva, dejando a un lado las sexualidades que se apartaban de este canon. La ciencia, así como legitimaría la sexualidad reproductora, caracterizó todos los posibles desvíos, implantando múltiples "perversiones". Es en este punto de la historia donde Foucault sitúa el nacimiento del homosexual como una nueva especificidad:

La categoría psicológica, psiquiátrica, médica, de la homosexualidad se constituyó el día en que se la caracterizó -el famoso artículo de Westphal sobre las "sensaciones sexuales contrarias" (1870) puede valer como fecha de nacimiento- no tanto por un tipo de relaciones sexuales como por cierta cualidad de la sensibilidad sexual, determinada manera de invertir en sí mismo lo masculino y lo femenino. La homosexualidad apareció como una de las figuras de la sexualidad cuando fue rebajada de la práctica de la sodomía a una suerte de androginia interior, de hermafroditismo del alma. El sodomita era un relapso, el homosexual es ahora una especie (Foucault, 1993: 31).

A partir de este estudio de Foucault, entendemos la homosexualidad como una categoría histórica producto del siglo XIX. De igual forma, obras como La crisis de la heterosexualidad (Guash, 2000), o Sexualidades occidentales (Aries et al., 1987), aportan una valiosa comprensión de la homosexualidad desde la historia: la sexualidad va íntimamente ligada al devenir de la sociedad en su conjunto. Para el caso particular de Colombia, una de las primeras investigaciones al respecto ha sido realizada por el historiador Walter Bustamante. En su libro Invisibles en Antioquia, 1886-1936: una arqueología de los discursos sobre la homosexualidad (2004), Bustamente parte de la revisión de expedientes del Archivo Histórico Judicial de Medellín -con juicios criminales que adelantaron las autoridades contra individuos acusados de ejecutar prácticas homosexuales-, la lectura de catecismos y manuales destinados a la educación y de algunos textos literarios de la época, así como de los debates en el Congreso, para considerar los discursos religiosos, jurídicos, educativos y médicos que pesaron sobre el homosexual a finales del siglo XIX y principios del XX en Colombia. Bustamente encuentra cómo sólo a comienzos del siglo XX la ley y el saber médico definieron como homosexual al sujeto que ama a otro de su mismo sexo, surgiendo 
por primera vez la categoría de homosexual con un saber y una verdad que lo soporta.

En el trabajo de Bustamante se evidencia la complejidad de abordar el tema de la homosexualidad. El autor lo explica desde la formas de saber y poder: si no se hace de esta forma -señala- se puede recabar en una descripción de los discursos sobre el homosexual desde una apología a la homofobia, ya que el sujeto homosexual no tiene voz y sólo es visibilizado por los dispositivos de poder, al ser los psiquiatras, policías, abogados, médicos y religiosos quienes hablan de él. Ya que en estas historias no aparece la voz del sujeto homosexual -pues "ha sido relegado al silencio, al ocultamiento y a la invisibilidad [...]" (Bustamente, 2004: 15)-, tendremos en cuenta que se hace indispensable analizar los discursos que desde el poder mencionan y construyen el imaginario sobre el sujeto homosexual en la Cartagena.

\section{El discurso científico: la patologización del homosexual}

La homosexualidad no siempre ha significado lo mismo a través de la historia, y las actitudes de odio y miedo contra los homosexuales no son un invento de la actualidad. El poder sobre la sexualidad se ha reacomodado con el paso de los años y ha producido discursos sobre lo que debe ser normal, natural y moralmente correcto. Es de esta forma como, desde el discurso religioso, se inventa al homosexual en calidad de "pecador": el llamado sodomita, el sujeto jurídico del pecado contra natura. Este discurso, sin embargo, perdería validez con el advenimiento de la modernidad: ya no era suficiente hablar de prácticas pecaminosas, ni de sodomía. Los nuevos saberes científicos serán, en adelante, los que produzcan "verdades válidas" para este nuevo contexto.

El sodomita podía cometer actos censurables condenados por la ley religiosa, calificados de contrarios a la naturaleza y perversión. No obstante, no se identificaba su conducta con un trastorno mental o con un desarreglo físico. Para llegar a una nueva categoría del homosexual como enfermo mental, el dispositivo tuvo que modificarse. La ciencia construyó un saber sobre la sexualidad, clasificó y categorizó lo normal y lo anormal. La psiquiatrizacion de la homosexualidad garantizó así un control social sobre los "desviados" mucho más sutil, un control en apariencia "positivo", ya que se trataba de corregir y arreglar lo que se suponía desviado y hacer un "bien" al sujeto afectado. 
De este cambio de la legitimidad del discurso religioso al discurso científico moderno es muestra el testimonio del ex-presidente Alfonso López Michelsen, quien en 1969, unos pocos años antes de ser Presidente de la Republica, escribió en el prólogo del libro El homosexualismo en el arte actual lo siguiente:

El aspecto ético del problema lo dejamos a quienes [...] consideren el homosexualismo como un pecado vitando. Para nosotros el problema es otro, creemos siguiendo el criterio de altas autoridades científicas, que el homosexualismo es una anomalía, y como tal, sujeta a las alternativas de su posible control o erradicación, al médico y al psiquiatra especializados en la materia corresponden las soluciones del caso y al estado que debe intervenir-científica y legalmente se sobreentiendeal respecto de esta sicosis que día a día aumenta [...] [sic] (Restrepo, 1969: 19).

De esta interesante cita se observan varios puntos. Primero, la posición que como liberal de la época asume el ex presidente: la homosexualidad, como problema ético, se lo deja a quienes la consideran un pecado (refiriéndose, por supuesto, a la religión). Segundo, atendiendo a la idea liberal del estado laico, se refiere a la homosexualidad como una "anomalía" de tipo médico, apelando a "altas autoridades científicas". Y tercero: hace un llamado a la intervención del estado para frenar esta "sicosis", recurriendo a la legitimidad científica moderna para el control efectivo de tal "desviación sexual".

Este nuevo sujeto patológico, al que se refiere López Michelsen, es también un sujeto más extendido; llega a localizarse en el vacío que los discursos morales y jurídicos habían dejado. Esa "ciencia", universal, valida sin excepciones, hace llegar el prejuicio y la homofobia allí donde la moral cristiana no se había establecido. Al promulgar la desviación sexual, las disciplinas científicas determinan también un tipo de "normalidad" (Llamas, 1998: 269).

La patología fue el dispositivo por cual se catalogó la homosexualidad como una categoría clínica: este tipo de "enfermos" eran atendidos por psiquiatras que intentaban hacerlos volver a la sexualidad "normal". Además, la patología implicó una caracterización del homosexual como inestable afectivamente, como un sujeto con un sistema nervioso degenerado, perturbado, narcisista, inmaduro, defectuoso en muchos aspectos de la vida y fijado en un momento infantil (Mandimore, 1998: 102). Por ejemplo, el Manual diagnóstico 
y estadístico de los trastornos mentales (DSM-I) ${ }^{3}$, publicado por la APA en 1952, clasificó la homosexualidad dentro de las llamadas "alteraciones sicopáticas de la personalidad", y posteriormente fue trasladada a la categoría de "otras alteraciones mentales no psicóticas” (De la Espriella, 2007).

Al respecto, el Doctor Álvaro Villar Gaviria, prestigioso médico psicoanalista que en varias ocasiones fuera presidente de la Asociación Colombiana de Psiquiatría, envió una carta al director de una obra de teatro cuya temática era la homosexualidad, en donde reconocía una persecución por parte la psiquiatría y el psicoanálisis a la homosexualidad. Un fragmento de la carta fue publicado junto al artículo que reseñaba el evento:

Le escribo bajo el impacto, que persiste después de haber ido anoche a presenciar Bent [...] pero cabría agregar lo concerniente a otra persecución que allí no se menciona y que apenas comienza a desaparecer: la procedente de la psiquiatría y del psicoanálisis de unas décadas atrás, que conozco no sólo por referencias sino porque tuve esos dos entrenamientos posteriores a la medicina, y aprendí eso como lo más natural: el homosexualismo como enfermedad mental y todas las variaciones de una conducta sexual ideal para las clases dominantes y naturalmente -no por coincidencia- para la iglesia católica, me fueron enseñadas como perversiones o aberraciones, los nombres médico-morales que equivalen exactamente a los correspondientes pecados de la religión (B.N.C ${ }^{4}$. Magazín Dominicial de El Espectador, 9 de febrero de 1985: 3, 6).

En las anteriores líneas Villar Gaviria reconoce su formación en la creencia naturalizada de la homosexualidad como "enfermedad mental" y muestra que era una creencia bastante extendida aún en 1985. Tal producción discursiva de la psiquiatría no se quedaría sólo en la opinión de algunos psiquiatras, sino que circuló socialmente y habría de reflejarse, por ejemplo, en un artículo de El Espectador que reseña una convención nacional sobre niñez, sexo y familia en el año de 1970. Uno de los temas tratados fue la homosexualidad:

\footnotetext{
${ }^{3}$ En psiquiatría, el Manual diagnóstico y estadístico de los trastornos mentales de la Asociación Psiquiátrica de los Estados Unidos (American Psychiatric Association) contiene una clasificación de los trastornos mentales y proporciona descripciones claras de las categorías diagnósticas, con el fin de que los clínicos y los investigadores de las ciencias de la salud puedan diagnosticar, estudiar e intercambiar información y tratar los distintos trastornos mentales. La edición vigente es la cuarta (DSM-IV). ${ }^{4}$ B.N.C. Estas siglas referencian la Biblioteca Nacional de Colombia.
} 
El estudio sobre el particular fue presentado por el médico Eduardo Laverde, el sociólogo Ángel Guarnizo y la antropóloga Ximena Pachón, quienes como recomendación proponen que se adelanten estudios sobre este problema y programas de acción específicos en orden a contrarrestarlos, toda vez que se presenta como inquietante reto a la estabilidad y normal progreso de la sociedad contemporánea (B.L.A.A. ${ }^{5} E l$ Espectador, 7 de Marzo de 1970: 11a).

Se recogen en esteartículo de la prensa nacional las conclusiones de un evento clausurado por el mismo presidente Carlos Lleras y su esposa, y confirma muy bien cómo en la opinión de médicos, sociólogos y antropólogos de la época la homosexualidad era un "problema", a tal grado que se pide adelantar estudios para contrarrestarlo, ya que es un "inquietante reto a la estabilidad y normal progreso de la sociedad contemporánea" (Ibíd.). Lo anterior evidencia cómo el discurso patologizante en Colombia se diseminó, reflejándose en la prensa e insertándose en el imaginario social.

La ciudad de Cartagena no fue la excepción al discurso patologizante sobre la homosexualidad. El estudio de este momento, sin embargo, es una tarea ardua que apenas inicia en medio de las dificultades de fuentes oficiales y de los muchos silencios que guarda la prensa. Por lo mismo, para estudiar en una primera instancia el proceso de patologización, se hace necesario recurrir a la fuente oral. Es así cómo, a través de entrevistas realizadas a psiquiatras en ejercicio para la década de los 70, trataremos de conocer de qué modo se construyó un sujeto "desviado" a partir de los discursos psiquiátricos.

Punto este de sumo cuidado, ya que a partir de 1973 se despatologizó la homosexualidad en los catálogos de clasificación de enfermedades mentales (DSM-IV), por lo que actualmente es engorroso para algunos psiquiatras confesar que alguna vez realizaron tratamientos psiquiátricos a homosexuales para curar su "desviación". Uno de los entrevistados, en el transcurso de esta investigación, fue el Dr. Cristian Ayola ${ }^{6}$, quien aclara que, aunque no trató a homosexuales con el fin de reconvertirlos, tuvo conocimiento de tratamientos psiquiátricos realizados a homosexuales en la ciudad de Cartagena:

\footnotetext{
${ }^{5}$ B.L.A.A. Estas siglas hacen referencia a la Biblioteca Luis Ángel Arango.

${ }^{6}$ Medico de la Universidad de Cartagena, con postgrado en Psiquiatría Biológica de la Universidad Oberta de Cataluña. Ex director del Hospital San Pablo y fundador de la clínica CEMIC. Durante 30 años ha ejercido como psiquiatra en Cartagena.
} 
[...] fundamentalmente estos eran sujetos de psicoterapias y de psicoanálisis [...] que era el método terapéutico aparentemente más efectivo que existía en la época. Posteriormente quedaron sin tratamiento. No había tratamientos, por lo menos biológicos, que yo conozca, no había para este trastorno. De pronto se escapa a mi conocimiento (Ayola, 2 de mayo del 2009).

El Dr. Ayola afirma que, efectivamente, para el llamado trastorno de la homosexualidad se utilizaban psicoterapias y psicoanálisis, si bien aclara que no eran sujetos de tratamientos biológicos. Esta respuesta nos permite inferir que en Cartagena si hubo psiquiatras que trataban la homosexualidad como parte de las muchas enfermedades mentales que atendían a diario. A continuación, mostraremos cómo el discurso patologizante sobre el homosexual apoyó y sustentó el discurso jurídico y, a la vez, cómo se reflejan estos discursos en la imagen del homosexual reproducida por la prensa.

\title{
Discurso jurídico: el homosexual como transgresor y delin- cuente
}

El dispositivo de poder sobre la sexualidad no se vale sólo de la legitimidad de la ciencia para construir sujetos anormales, sino que, como afirma Ricardo Llamas, "los poderes civiles tienen un papel destacado en el control de los afectos o los placeres considerados ilegítimos" (1998: 30). Ello se evidencia, por ejemplo, en el Código Penal colombiano que regía en el periodo de estudio. El capítulo IV, titulado "De los abusos deshonestos", reza así:

\begin{abstract}
Artículo 323. El que ejecute sobre el cuerpo de una persona mayor de diez y seis años un acto erótico-sexual, diverso del acceso carnal, empleando cualquiera de los medios previstos en los artículos 317 y 320, estará sujeto a la pena de seis meses a dos años de prisión. En la misma sanción incurrirán los que consuman el acto sexual homosexual cualquiera que sea su edad (Código Penal, 1937. Las cursivas son nuestras).
\end{abstract}

Clara tipificación de la pena sobre el homosexual que muestra la imbricación de los poderes civiles, religiosos y científicos coincidentes en cuanto a la heterodoxia sexual se refiere. En este orden de ideas, la ley actúa confirmando el prejuicio, "considerando que los homosexuales no pertenecen al ámbito de la ciudadanía y no pueden tener un reconocimiento que no sea el condenatorio" (Llamas, 1998: 246). El hecho de que en el Código Penal colombiano regente en ese momento apareciera la homosexualidad con una pena 
especificada es un ejemplo de la lectura exclusivamente heterosexual que la legislación daba a la sociedad y a los afectos en el país.

A pesar de que la elaboración teórica del discurso jurídico no fue tan abundante y original en conceptos, como si lo fue el discurso patologizante psiquiátrico, éste era el aparato que se encargaba del control social efectivo sobre los homosexuales (Llamas, 1998: 247). La ciencia construyó la "verdad" sobre la cual se cimentó el poder civil, y éste construyó a su vez los espacios físicos de exclusión, ya que desde la legislación se veía a la "sociedad" como víctima y a la moralidad como bienes jurídicos que debían ser protegidos (Llamas, 1998). Es en este sentido que, además de la construcción de la desviación hecha por la psiquiatría, el discurso jurídico suma la idea de transgresión y delincuencia. De lo anterior es muestra una nota de prensa del año 1973 publicada en Cartagena, que se refiere al asesinato de un joven llamado Gabriel Ballesteros y de las posibles hipótesis sobre su muerte:

En torno a su muerte se ha tejido una serie de versiones, entre ellas las [sic] de que la pasión puede ser uno de los móviles del homicidio.

Se ha dicho que Gabriel Ballesteros, aun cuando andaba con jóvenes de buena presencia siempre despertaba sospechas sobre sus actitudes morales y andanzas.

De otra parte se ha especulado que el occiso al parecer tenía amistades con elementos homosexuales, presumiéndose que hubiere sido víctima de uno de estos enfermos sexuales, esta última hipótesis es estudiada por las autoridades secretas debido a que la víctima nunca fue vista en compañía de jovencitas sino de esta clase de sujetos [...] [sic] (A.P.U. El universal, 17 de noviembre de 1973: 5)7.

El anterior artículo es revelador. Nos dice, por ejemplo, que el sólo hecho de que el joven asesinado tuviera amigos homosexuales convertía a estos en principales sospechosos, lo cual revela lo sumamente arraigado que estaba en el imaginario social la "anormalidad" y la "peligrosidad" de estos sujetos. En esta época, con toda la carga negativa que recaía sobre el sujeto homosexual, lo más lógico era pensar que, siendo "enfermos sexuales", fueran los principales sospechosos de efectuar un crimen.

${ }^{7}$ A. P. U. Estas siglas referencian el Archivo del Periódico El Universal. 
Por otro lado, la ausencia de disposiciones legales especificas que criminalizaran los placeres y afectos entre personas del mismo sexo, y la imposibilidad de localizar la multiplicidad de expresiones homoeróticas que abundan en cualquier sociedad, los sistemas judiciales hicieron de determinados delitos, como el escándalo público y otros elementos, formas de represión privilegiadas. Otra columna de prensa del año 1977 en la ciudad de Cartagena reseña así la detención de varios sujetos homosexuales:

\begin{abstract}
Varios sujetos fueron detenidos en el día de ayer por agentes secretos de la policía bajo la sindicación de ser pervertidos sexuales los cuales fueron sorprendidos en el momento en que ejecutaban actos anormales en lugares públicos. Los detenidos ingresaron a la cárcel de San Diego a la orden del funcionario de competencia.
\end{abstract}

\title{
DETENIDOS
}

Se trata de los sujetos Francisco Coavas Ortiz [...] y Alberto Salazar Castellar [...] quienes fueron sorprendidos en actos contra la moral en las inmediaciones del Sena. [...] (A.H.C. Diario de la costa, 20 de julio de 1977: 9) ${ }^{8}$.

Observamos en el citado aparte de prensa la retórica discursiva utilizada para referirse al sujeto homosexual. Se reitera en este artículo que se ejecutaban "actos anormales", concepto totalmente psiquiátrico, lo cual apoya nuestro argumento de que en el imaginario popular la patologización del homosexual fue una metáfora social que se diseminó en la cotidianidad de la ciudad de Cartagena, teniendo en cuenta todos los adjetivos que acompañaban la categoría homosexual en las referencias de prensa. De igual forma, semejante argumento es acompañado por el calificativo de "actos contra la moral". En este sencillo ejemplo obsevamos cómo en una detención los argumentos eran de índole moral y científico, argumentos que estaban cotidianizados, normalizados y naturalizados, muy a pesar de que estos sucesos son tomados de un periódico del año 1977. Cuatro años después de que se despatologizara la homosexualidad se le continuaba etiquetando como perversión sexual.

\section{Conclusiones}

Podemos anotar que sí existió un discurso patologizante sobre la homosexualidad en Cartagena que se revela sutilmente, que permea

8. H. C. Estas siglas hacen referencia el Archivo Histórico de Cartagena. 
los discursos jurídicos y se manifiesta en la imagen construida por la prensa sobre los sujetos homosexuales. Era de uso extendido la categoría de homosexual con una fuerte carga de conceptos psiquiátricos, lo cual apoyóy legitimóla exclusión, así como diferentes tipos de controles sociales ejercidos por la justicia.

Discurso patologizante que, indudablemente, llena cada uno de los adjetivos que acompañan las referencias al homosexual, incluso mucho después del año 1973, cuando fue despatologizada, evidenciando que, aunque se dieran cambios en el seno de la ciencia y su discurso, la imagen del homosexual como anormal ya se había anclado en el imaginario social, y de esa misma manera es representada por la prensa. Por otro lado, las herramientas jurídicas para este periodo sin duda ejercen los controles efectivos contra el homoerotismo masculino en Cartagena.

Los discursos patologizantes y represores sobre el homosexual construyeron y reafirmaron diversas formas de exclusión, haciendo que hoy en día se haya naturalizado y normalizado la discriminación, cumpliendo con la función de un dispositivo de poder sobre la sexualidad que construyó cuerpos normales y anormales todavía grabados en los imaginarios de la ciudad

\section{Bibliografía}

\section{Fuentes primarias}

El Espectador

El Universal

Diario de la Costa

\section{Fuentes secundarias}

Ariès, P., Béjin, A. et al. (1987). Sexualidades occidentales. Barcelona: Paidós.

Bustamante, W. A. (2004). Invisibles en Antioquia, 1886- 1936: una arqueología de los discursos sobre la homosexualidad. Medellín: La Carreta.

Código Penal. (1937). Bogotá: Imprenta Nacional.

De la Espriella, R. (2007). "Homofobia y psiquiatría”, Revista Colombiana de Psiquiatría, vol. 36. º.4, Bogotá.

Foucault, M. (1993). Historia de la Sexualidad: La Voluntad de Saber. México: Siglo XXI, vol. I.

Gabriele, A. (2003). "Notas para un análisis de las categorías de normalidad y patología en el discurso psiquiátrico-político". II Congreso 
Interoceánico de Estudios Latinoamericanos, Mendoza (Argentina), 11 al 13 de septiembre.

Gamboa, I. (2006). "La constitución de trastornos sexuales en la psiquiatría”, Diálogos, Universidad de Costa Rica, vol. VII.

Guash, 0. (2000). La crisis de la heterosexualidad. Barcelona: Laertes.

Lamas, M. (1995). “Cuerpo e identidad”. En Arango, L. et al (Comp). Género e identidad: ensayos sobre lo femenino y lo masculino. Bogotá: TM editores.

Llamas, R. (1998). Teoría torcida: prejuicios y discursos en torno a la homosexualidad. Madrid: siglo XIX.

Mandimore, F. Mark. (1998). Una historia natural de la homosexualidad. Barcelona: Paidós.

Muñoz, D. (2006). "Sexualidades ilegitimas, biopolitica heterosexista y política de reconocimiento", Nómadas, Bogotá, Universidad Central.

Restrepo, P. (1969). El homosexualismo en el arte actual. Bogotá: Tercer mundo. 
